\title{
Training of Health Promotion Agents: An Intervention in the Baoulé Community of Kongodékro (North- Central Côte d'Ivoire)
}

\author{
Anoua Adou Serge Judicaël* \\ Alassane Ouattara University, Ivory Coast
}

*Corresponding author: Anoua Adou Serge Judicaël, Anthropology and Sociology Department, Alassane Ouattara University, Ivory Coast.

Received Date: October 22, 2019

Published Date: October 29, 2019

\begin{abstract}
The training of health promotion agents meets a healthy expectation in front of the multiplicity and complexity of health problems in Côte $d^{\prime}$ Ivoire. In order to establish the mechanism of behavioral change in the population, a simulation put the auditors in a situation of intervention in the village of Kongodékro. This process helped to identify priority problems and solutions, to plan activities and to define follow-up procedures, evaluate and archive reports. This scenario is a unique experience to strengthen and enhance the skills and values of community health auditors.
\end{abstract}

Keywords: Transformative action; Community approach; Capacity strengthen; Health.

\section{Introduction}

Public health problems in Côte d'Ivoire are dominated by infectious and parasitic diseases, the most important of which are malaria, diarrheal diseases and pneumonia. Children and pregnant women are the most vulnerable and affected populations [1]. To this alarming situation is added the progression of chronic and degenerative diseases. From this complex health situation comes a life expectancy backwards. How can the vast majority of the population benefit from a viable health at a lower cost and reduce the burden of disease in Côte d'Ivoire? The relevant assumption for a sustainable health for the entire population is the establishment of a health promotion system anchored in lifestyle. It is an immersion framework in which the different social strata of the population take charge of their own health by acting on the risk factors [2]. This dynamic health framework requires coordinated action [3]. In this context, nurses, midwives and doctors are recruited from the community health training program at Alassane Ouattara University in Bouaké. They are trained in a two-year cycle with the skills of health promotion agents. It is for these auditors to strengthen their ability to support the community in order to define the priorities of health problems, solutions, action plans to change behavior. To be convinced, a simulation aimed at putting auditors in a field intervention situation took place in the village of Kongodékro. In reality, it is about implementing the development and implementation of a community health project. This intervention is understood as an emulation based on the values, knowledge and know-how of stakeholders beneficial to the health of populations. This dynamic training process is based on a particular approach.

\section{Implementation}

The immersion of auditors in the community recommended a scenario. The stages of the scenario are the preparatory phase of the field, the community diagnosis, the community feedback workshop and the presentation of the implementation reports. The purpose of these activities is to master the participatory and community approach to strengthen the skills of health promotion agents.

\section{The Preparatory Phase of the Field}

This phase took place a week before the release. It revolves around the prospecting mission, the development of the administrative protocol and the development of tools for community diagnosis. 


\section{The prospecting missions}

The prospecting mission was entrusted to a group of auditors. The village of Kongodékro in north-central Côte d'Ivoire has been identified as the investigative community. Physical contact with the various local, political, administrative and customary authorities has led to good community mobilization, a guarantee of success for the community health project. Subsequently, this community visit also led to the identification of all the health problems with the community and staff at the local health center. There is a high rate of consultation related to malaria, diarrheal diseases in children, acute respiratory failure, inadequate antenatal care, unaided deliveries, and unsanitary conditions. From the exchanges, the epidemiological profiles of the locality in question were identified and validated by seeking the reason of their priority character by the local community. It is therefore the low use of maternal services of Kongodékro by pregnant women and the insalubrity. From the identification and validation of the priority problems, it is necessary to move on to the administrative protocol.

\section{The administrative protocol}

This administrative approach was the work of a group of auditors. As part of the field intervention, it consisted of informing local, political, administrative or departmental, and customary authorities of the scientific intent of the research project through correspondence issued by the academic authority. This administrative procedure leads to the development of tools for community diagnosis.

\section{Development of community diagnostic tools}

The phase of identification and development of community diagnostic tools was organized with all the auditors. Two working groups were formed to address each one a priority health issue. From a research-action perspective, the following data collection techniques were retained: the review of birth registers, the focus group, the historical profile and the field visit. All these tools have been validated under the supervision of the supervisors. It is now a question of how the actual community diagnosis is conducted in the field.

\section{Community Diagnosis}

It marks the effectiveness of investigations in the field. It relies on a ritual of civility prior to the implementation of the collection scenario and data analysis.

\section{The ritual of civility}

The ritual of civility was here a moment of communion of the entire delegation with the local chieftaincy, the health workers and the community. During this protocol of use, exchanges of news, the knowledge with the notability, the clarification of the object of the mission and the course of the range of the activities furnished these moments. As a result of this sharing, a favorable opinion was issued by the chieftaincy and the community, as well as thanks by the delegation of auditors for their agreement. A libation made by the chief of the village of kongodékro thus symbolically testified to the community's support for this community intervention. As a result of this usage protocol, the data collection scenario was put in place.

\section{Setting up the data collection and analysis scenario}

The implementation of the data collection and analysis scenario put auditors and the community in contact. Its objective was to first have a collection of information focused on the priority problems from the data collection tools developed and then a fact by bringing the various stakeholders to explain more information collected. This modality of organization of the facts led to the restitution workshop in the community.

\section{The Restitution Workshop in the Community}

The restitution workshop in the community sparked an animation between the auditors and the community. Its objective has been to identify priority issues, identify priority solutions, plan activities, assign tasks, roles and responsibilities in the implementation and definition of monitoring and evaluation modalities archiving of reports by the community. At the end of this planning, all of this implementation needs to be written into reports.

\section{Presentation of Implementation Reports}

The implementation reports include a mission report and a scientific report. They were the subject of group work, exchange, evaluation and lessons learned by auditors. The mission report concerned the description of the entire process of the intervention. It has an administrative character and has been addressed to the administrative, customary and community authorities and local health workers. The scientific report is seen as a document for implementing community health. It has a scientific character and it has been addressed to the academic authority in order to highlight the results in terms of impact in the short and medium term and to give a scientific opinion on the problems identified, the results and make recommendations.

\section{Results}

The results to be observed are marked by a scientific character. They derive from the implementation of community health through the community diagnosis and the action matrix. They are perceived at two levels of problematic situation: the low use of maternity services of Kongodékro by the pregnant women and the insalubrity in said village.

The problem of the Use of Maternity Services at the Kongodékro Health Center by Pregnant Women

\section{Inadequate antenatal care}

The analysis of the low use of maternity services has led the community to identify several sensitive risk factors and possible solutions.

The first priority problem was the lack of antenatal care. Faced with this situation, two priority solutions have been proposed by the community. 
Initially, it would involve more involvement of spouses in the prenatal consultations of their wives. To do this, the social actors committed to the task would be the community health worker and the midwife. These should respectively provide door-to-door outreach in the village through megaphone and couple counseling during prenatal consultations at the health center from the picture boxes. In terms of evaluation, the number of spouses sensitized and the number of spouses accompanying their wives in prenatal consultation would make it possible to assess the process and the effectiveness of this action.

In a second step, it would be a question of sensitizing the pregnant women to respect the appointments of the prenatal consultations. The midwife, community health worker and spouses would be available, respectively, to provide counseling, doorto-door outreach, and the search for the lost to the image box, megaphone, and follow-up prenatal consultation records seen in this activity. In the medium and long term, the number of pregnant women sensitized on the respect of prenatal consultations and the antenatal consultation rate greater than or equal to four would be indicators of the monitoring and effectiveness of this action.

\section{Low rate of assisted deliveries}

The second priority problem was the low birth rate in the Kongogodekro maternity ward. In this case, the community has suggested four priority solutions.

First, there was the reorganization of the maternity service, including permanency and care. This activity would be the responsibility of the midwife through service meetings. For this, the provision of office and an equipped guard room would be among other laudable means. 24 hours a day, seven days a week the Kongodékro Health Center, as well as one hundred percent (100\%) of the hours of duty and guards each month should reflect the reality of implementation. express the achievement of this activity.

Secondly, the good collaboration between the health staff, particularly the midwife and the community, was emphasized. The actors willing to do this task would include the midwife and the entire community. The organization of exchange meetings with the community using material means such as the megaphone, chairs and a meeting room as well as home visits should be useful to the realization of this solution. Thus, the number of home visits made, i.e. four visits by the midwife a month, and two midwife exchanges with the community per month should express the achievement and effectiveness of this action.

Third, the construction of housing for the midwife has been accepted. This activity would be the responsibility of the people and the officials of the village. In this initiative, it would be a question of making a plea to the administrative authorities and to encourage the mobilization and the participation of the village community. To do this, letters to the mayor and the regional council and the contribution of the community would be the means available. In this process, the monitoring indicators would be the written letters sent to the town hall and the regional council and then the levy within the community is ten percent $(10 \%)$ of the project budget. As for the efficiency indicator, it would be observable through the number of members of the community to have contributed at a given period and the external contributions obtained from the decentralized communities.

This community diagnosis and the planning of activities in the field of reproductive health leads to a glimpse of the problems and solutions identified in the case of environmental health.

\section{The problem of insalubrity in the village of Kongodekro}

In the analysis of the unsanitary situation, the priority problems relating to the lack of initiative of sanitary activities and the proliferation of wild garbage dumps were similarly identified, and the means to act on these problems were identified.

\section{Lack of initiative of safety activities}

Regarding the problem of lack of initiative of the health activities, two priority solutions were admitted by the community.

First, the planning of health activities was noted. It should be carried out by community leaders and health workers. They would be required to hold exchange meetings to develop safety implementation activities. To do this, notebooks, pens and computers would serve as work media. All in all, the holding of four exchange meetings and the provision of a business plan should convince the realization of this solution.

The cleaning of the living environment was then observed. This activity would be the work of the young men and women of the village from operation brooms, garbage collection, cleaning open septic tanks and installation of garbage cans. These property actions would require, as means, wheelbarrows, gloves, machetes, rakes, dabas, shovels, brooms, mufflers. Moreover, two days of sanitation every month would testify to the effectiveness of this activity.

\section{Proliferation of wild garbage dumps}

Concerning the problem of proliferation of wild garbage dumps, two priority solutions were chosen by the community.

In the first place, the use of household garbage cans would affect all households. It would be for people in each family to throw garbage in the garbage. These would identify with used buckets and plastic bins. Their presence in the majority of households should express the realization of this solution.

Secondly, the creation of dumps for the village would mobilize the whole community. The goal would be to build a site outside the village for garbage collection. If necessary, machetes, shovels, picks and wheelbarrows would be the means of action. The presence of an operational dump is an indicator of the achievement of this activity.

In the light of the real problems that arise, and the appropriate actions defined in this matrix of actions of the village of Kongodékro, what lessons can be learned from this community intervention? 


\section{Implications}

The critical examination of the community intervention in Kongodékro village revealed the strengths and weaknesses of this activity.

The forces concerned legal authorization, social legitimacy, the availability of social actors (health professionals \& population), the immersion of facilitators in the community and the use of appropriate tools for community diagnosis.

The weaknesses were related to the absence of some members of the community during the restitution, to the absence of investigators trained for the collection of information, to the absence of cross-cutting questions put to the interlocutors in the sense of the modalities of solutions, the omission of certain collection tools such as the seasonal calendar and the Venn diagram essential for the planning of activities.

Ultimately, it is important to remember that although the steps in the development of a community health project are defined, none of them is fixed. The methodology must be rigorously respected. The development of a community health project is essentially based on the community, i.e. its total involvement under the control of a facilitator.

\section{Conclusion}

This intervention in the community of kongodekro allowed the immersion of community health auditors. In this context, the preparatory phase of the field, the community diagnosis and the restitution workshop in the community were the steps of the process. At the end of these stages, priority problems were identified, and action plans were defined. This scenario is a unique experience to strengthen and enhance the skills and values of community health auditors. The community approach, as exciting as it is painful, requires the openness, availability and humility of health promotion agents. This appears as a guarantee of access to local knowledge and practices, behavioral changes within the population and support for the control of their own health.

\section{Acknowledgement}

None.

\section{Conflict of Interest}

Author declare no conflict of interest.

\section{Refernces}

1. (2016) Ministry of Health and Public Hygiene (MSHP) National Health Development Plan 2016-2020. Republic of Côte d'Ivoire.

2. Kerouedan D, Audibert M (2015) Health Development in Francophone Africa.

3. Jourdan D (2019) Renewing the framework of health promotion: the research and reflection axes. Context and re-definition of the field. In: A seminar to re-question the framework of health promotion, Clermont Auvergne University, France. 